\title{
Aneurisma de artéria femoral superficial roto: relato de caso e revisão de literatura
}

\author{
Ruptured superficial femoral artery aneurysm: \\ case report and literature review
}

Alexandre Faraco de Oliveira ${ }^{1}$, Horácio de Oliveira Filho ${ }^{2}$

\section{Resumo}

$\mathrm{O}$ aneurisma de artéria femoral superficial é um evento raro que costuma manifestar-se em pacientes com idade avançada, frequentemente associado a outros aneurismas, periféricos ou de aorta abdominal. $\mathrm{O}$ caso relatado refere-se a um paciente cujo aneurisma de artéria femoral superficial se apresentou roto e associado a um aneurisma de aorta abdominal. Foi submetido à revascularização cirúrgica fêmoro-poplítea mediante prótese de politetrafluoretileno, com sucesso. A revisão da literatura demonstra que embora haja uma tendência à manifestação aguda da doença, principalmente como ruptura, os resultados com o tratamento cirúrgico são excelentes. ruptura.

Palavras-chave: Artéria femoral superficial, aneurisma roto,

\section{Introdução}

$\mathrm{O}$ aneurisma da artéria femoral superficial é uma condição rara. Como lesão isolada, já foi descrito como responsável por apenas $0,5 \%$ dos aneurismas periféricos; menos frequente ainda tende a ser sua apresentação aguda, seja devido à ruptura local ou à trombose do aneurisma ${ }^{1-3}$.

Neste trabalho, relatamos um caso de ruptura de aneurisma da artéria femoral superficial isolado seguido da correção cirúrgica convencional e descrevemos a revisão bibliográfica.

\section{Relato do caso}

Paciente de sexo masculino, 87 anos, trabalhador rural, tabagista. Foi recebido em consultório médico referindo dor e aumento de volume na coxa direita com evolução de 48 horas.

Antecedentes: o paciente referia esta como sua primeira consulta médica e negava qualquer queixa prévia rela-

\begin{abstract}
Superficial femoral artery aneurysm is rare and usually occurs in elderly patients, often associated with peripheral or abdominal aortic aneurysms. In the reported case, the patient presented with a ruptured superficial femoral artery aneurysm associated with an abdominal aortic aneurysm. A successful femoropopliteal bypass was performed using a polytetrafluorethylene graft. Literature review shows that, despite the tendency for acute onset of the aneurysm, primarily with a rupture, surgical results are excellent.
\end{abstract} ture.

Keywords: Superficial femoral artery, ruptured aneurysm, rup-

cionada aos membros inferiores, edema ou claudicação. Negava histórico de hipertensão arterial e diabetes melitus. Ao exame físico, apresentava-se descorado, taquicárdico (110 bpm) e hipotenso (100 x $60 \mathrm{mmHg}$ ). Membros inferiores: à esquerda, sem qualquer alteração aparente, com todos os pulsos palpáveis e de amplitude reduzida nas pernas; à direita, equimose em raiz de coxa estendendo-se para o escroto, abaulamento a partir do ligamento inguinal até o terço distal da coxa, pulsátil, com frêmito em face anterior, terço médio, pulso femoral presente e demais ausentes.

Foi realizado eco-Doppler colorido arterial em membro inferior direito que revelou volumoso aneurisma de artéria femoral superficial em terço médio (Figura 1), medindo no maior diâmetro transverso $3,45 \mathrm{~cm}$, com fluxo turbulento e hipocinético em seu interior e ruptura em face medial (Figura 2). Exames laboratoriais revelaram acentuada anemia (Hb: 6,7; Ht: 20,4).

1. Cirurgião vascular. Ultrassonografista vascular. Professor, Curso de Medicina, Universidade do Planalto Catarinense (UNIPLAC), Lages, SC.

2. Cirurgião vascular. Especialista em Angiologia e Cirurgia Vascular, SBACV.

Não foram declarados conflitos de interesse associados à publicação deste artigo.

Artigo submetido em 27.10.08, aceito em 12.06.09.

J Vasc Bras. 2009;8(3):283-288.

Copyright (C 2009 by Sociedade Brasileira de Angiologia e de Cirurgia Vascular 


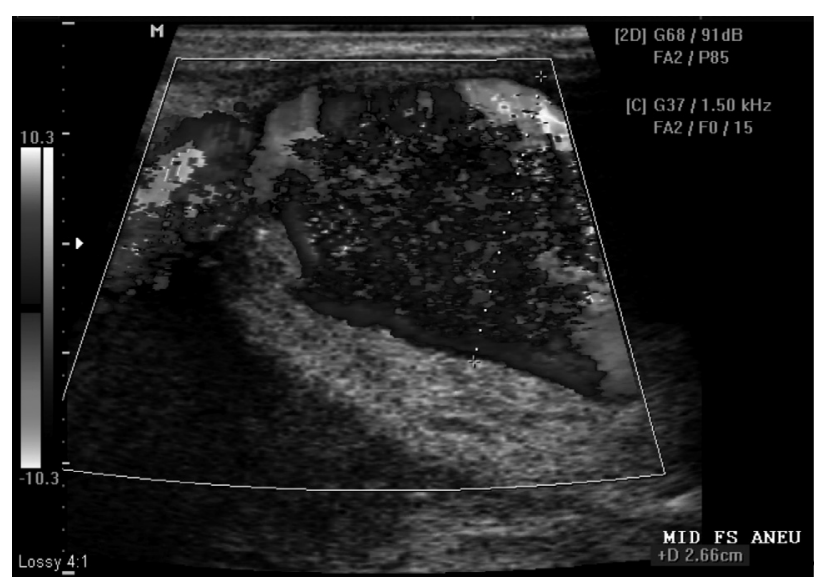

Figura 1 - Dúplex colo proximal e fluxo em aneurisma de artéria femoral superficial

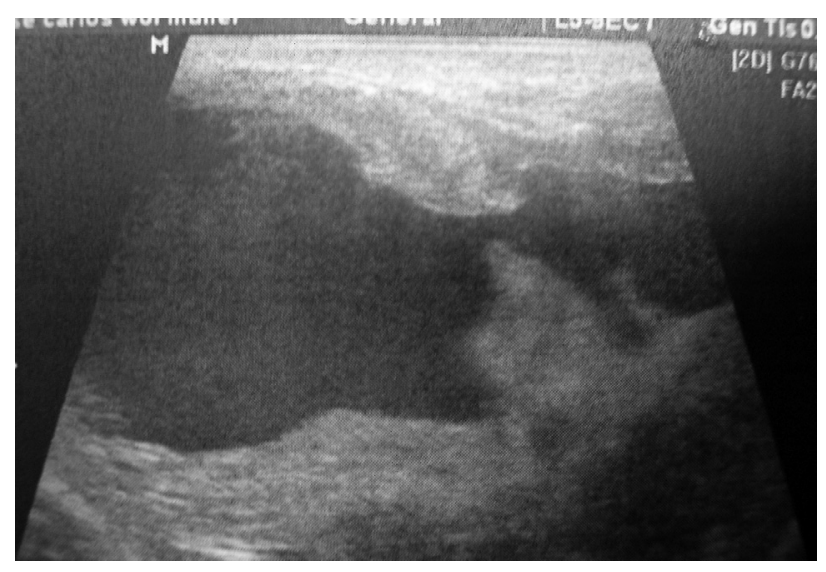

Figura 2 - Dúplex local de ruptura do aneurisma de artéria femoral superficial

Submetido o paciente a cirurgia imediatamente, constatou-se volumoso aneurisma de artéria femoral superficial isolado roto com grande quantidade de sangue junto aos tecidos musculares e subcutâneos, principalmente em face medial (Figura 3). Foi realizado o reparo através de revascularização fêmoro-poplítea com a utilização de prótese (politetrafluoretileno $8 \mathrm{~mm}$ ), anastomose término-terminal tanto proximal quanto distal e ressecção parcial do saco aneurismático (Figura 4). A veia autóloga safena magna não foi utilizada por apresentar-se afilada e fibrosada.

No controle 30 dias após a cirurgia, o paciente não relatou qualquer queixa e deambulava sem restrições. Realizado dúplex arterial, revelou perviedade da prótese sem outros aneurismas nos membros inferiores. O paciente apresentava aneurisma da aorta abdominal suprarrenal medindo $5,76 \mathrm{~cm}$ no maior diâmetro. $\mathrm{O}$ exame do material

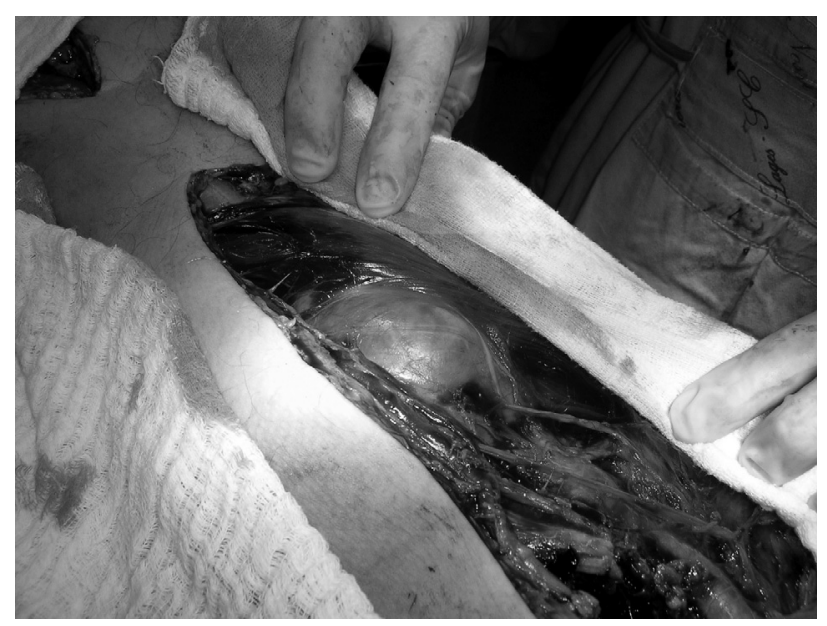

Figura 3 - Transoperatório, dissecção do saco aneurismático

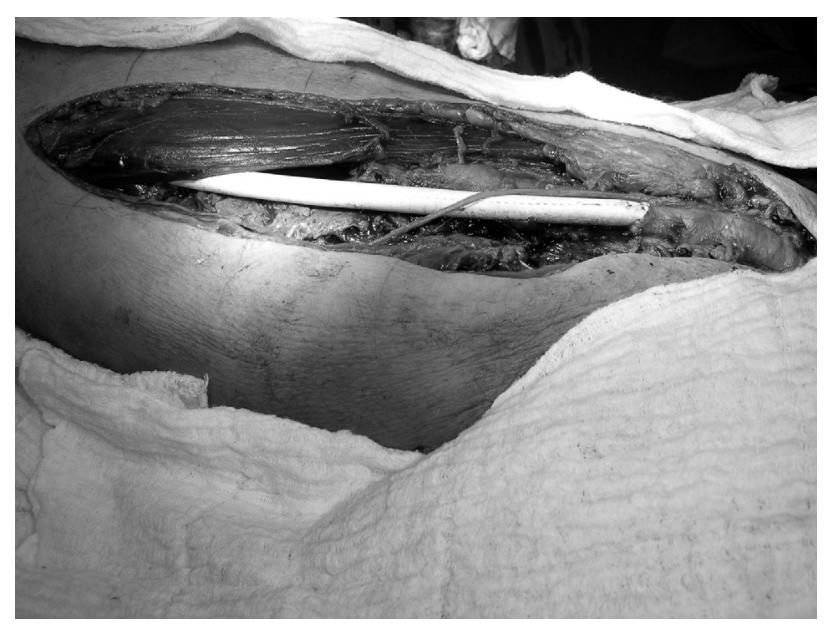

Figura 4 - Transoperatório, aspecto final da revascularização com politetrafluoretileno

recolhido da parede aneurismática não apresentou crescimento bacteriano, nem alterações na estrutura da artéria que sugerissem doenças outras que não a arteriosclerose.

\section{Discussão}

Através da pesquisa em banco de dados via internet, pudemos constatar a raridade da doença em face do número reduzido de casos relatados, e poucas séries.

O primeiro caso relatado de ruptura de aneurisma de artéria femoral superficial data de 1975 e já refere esta situação como rara e frequentemente associada com outros aneurismas. Assinala-se que o tratamento com revascularização fêmoro-poplítea, preferencialmente com veia autóloga, apresenta bons resultados nestes casos ${ }^{3}$. 
Em 1981, Kremen et al. ${ }^{4}$ publica uma série de sete aneurismas em seis pacientes e revisa a literatura existente. Conclui que a doença acomete pacientes com idade e aterosclerose avançadas e tende a se apresentar com efeito de massa local e ruptura. Nesta série, relata outros aneurismas periféricos em $50 \%$ dos pacientes e aneurisma de aorta abdominal em 33\%. Os autores consideraram a exclusão do aneurisma associada à revascularização fêmoro-poplítea com veia autóloga o tratamento adequado. Concluem com a indicação de reparo cirúrgico a fimm de evitar a tendência à ruptura e eventual embolia ${ }^{4}$. Diversos autores em relatos de casos isolados corroboraram as conclusões da série anterior, tanto no que se refere à apresentação da doença quanto ao tratamento cirúrgico ${ }^{5-9}$.

Em 1992, Rigdon \& Monajjem ${ }^{10}$ relata dois casos com apresentações e evoluções diversas: uma ruptura seguida de revascularização e salvamento do membro e uma trombose seguida da perda do membro mesmo com o tratamen- to cirúrgico. Em sua revisão de 17 casos em 14 pacientes, o autor refere que em $65 \%$ dos casos o aneurisma se manifestou de forma aguda (ruptura, 35\%, trombose, $18 \%$ e embolização distal, 12\%). Não houve mortalidade perioperatória, e o salvamento do membro ocorreu em $94 \%$ dos casos. $\mathrm{O}$ aneurisma de aorta abdominal estava presente em $40 \%$. O autor ressalta a importância da investigação para determinar possíveis etiologias da doença - infecciosa (sífilis, etc.), inflamatória, imunológicas, colagenoses - antes de considerá-la aterosclerótica ${ }^{10}$.

Embora o tratamento convencional com a revascularização fêmoro-poplítea, preferencialmente com veia autóloga, tenha apresentado excelentes resultados, outras técnicas podem ser utilizadas. Dighe \& Thomas ${ }^{11}$ relatam um caso de aneurisma roto de artéria femoral superficial em um paciente com arteriopatia conhecida já submetido a tratamento cirúrgico de outros aneurismas, cuja parede da artéria poplítea se apresentava doente, sem condições de

Tabela 1 - Relatos e séries sobre aneurisma isolado de artéria femoral superficial

\begin{tabular}{|c|c|c|c|c|}
\hline Autor & AFS & Apresentação & Evolução & Outros aneurismas/número de pacientes \\
\hline Hardy \& Eadie ${ }^{17}$ & 01 & Eletivo & + & Não \\
\hline Ormstad \& Solheim ${ }^{3}$ & 01 & Ruptura & + & Sim \\
\hline Kremen et al. ${ }^{4}$ & 07 & Ruptura $^{3} /$ Trombose $^{3} /$ Eletivo $^{1}$ & + & $\operatorname{Sim} / 02$ \\
\hline Celi et al. ${ }^{18}$ & 01 & Eletivo & + & Não \\
\hline Cielik et al. ${ }^{8}$ & 01 & Ruptura & + & Sim \\
\hline Parra et al. ${ }^{7}$ & 01 & Ruptura & + & Não \\
\hline Bonelli et al. ${ }^{6}$ & 02 & Ruptura / Ruptura & + & Não \\
\hline Mayall et al. ${ }^{19}$ & 02 & Eletivo / Eletivo & + & Não \\
\hline Rigdon \& Monajjem ${ }^{10}$ & 02 & Ruptura / Trombose & $+/-$ & Não \\
\hline Vasquez et al. $^{20}$ & 01 & Trombose & + & Não \\
\hline Atallah et al. ${ }^{21}$ & 02 & Ruptura / Ruptura & + & $\operatorname{Sim} / 01$ \\
\hline Farinon et al. ${ }^{5}$ & 01 & Ruptura & + & Não \\
\hline Diethrich $^{12}$ & 01 & Eletivo & + & Não \\
\hline Dimakakos et al. ${ }^{22}$ & 02 & Ruptura / Ruptura & + & $\operatorname{Sim} / 01$ \\
\hline Jarrett et al. ${ }^{15}$ & 13 & Trombose $^{6} /$ Eletivos $^{7}$ & $+11 /-02$ & $\operatorname{Sim} / 10$ \\
\hline Coppin et al. ${ }^{23}$ & 01 & Ruptura & + & Não \\
\hline Galindo et al. ${ }^{9}$ & 01 & Ruptura & + & Não \\
\hline Honjo et al. ${ }^{24}$ & 01 & Eletivo & + & Não \\
\hline Siani et al. ${ }^{25}$ & 01 & Ruptura & + & Não \\
\hline Pulcini et al. ${ }^{26}$ & 01 & Ruptura & + & Não \\
\hline Dighe \& Thomas ${ }^{11}$ & 01 & Ruptura & + & Sim \\
\hline Relato atual (2009) & 01 & Ruptura & + & Sim \\
\hline Total $(\%)$ & 45 & $\begin{array}{c}\text { Ruptura }^{20}(45) \\
\text { Eletivo }^{14}(31) \\
\text { Trombose }^{11}(25)\end{array}$ & $\begin{array}{c}+42(93) \\
-03(07) \\
\text { Mortalidade } 0\end{array}$ & $\begin{array}{l}\text { Não } 27 \text { (60) } \\
\text { Sim } 18 \text { (41) }\end{array}$ \\
\hline
\end{tabular}

AFS = aneurisma femoral superficial; - = perda do membro; + = salvamento do membro. 
receber um enxerto; foi realizada a ligadura proximal e observada perfusão distal adequada via circulação colateral. O paciente foi tratado com a simples ligadura, sem que houvesse uma revascularização posterior, e evoluiu com o salvamento do membro ${ }^{11}$.

Nos últimos anos, a difusão dos métodos endovasculares tem permitido o tratamento para pacientes com risco cirúrgico elevado, com menor morbimortalidade. Isto pode beneficiar substancialmente os pacientes que se encontram na sétima e oitava décadas de vida. O uso de stents recobertos para tratamento de aneurisma de artéria femoral superficial já foi realizado com sucesso, ainda que a patência destes enxertos a longo prazo não esteja definida ${ }^{12}$. Relatos recentes utilizam stent recoberto para reparar rupturas espontâneas de artéria femoral superficial (sem doença aneurismática), mas a fasciotomia descompressiva pode ser necessária devido ao edema compartimental ${ }^{13,14}$.

O pequeno número de casos dificulta qualquer conclusão a respeito da doença; entretanto, tem se questionado a real etiologia aterosclerótica dos aneurismas de femoral superficial devido a sua associação com outros aneurismas. Também a real incidência da doença pode estar mascarada, pois a angiografia tende a não revelar o aneurisma caso este se apresente ocluído ${ }^{9,15}$. As revisões sobre o tema sugerem que o aneurisma de artéria femoral superficial tende a se comportar mais como um aneurisma aórtico (ruptura) do que como um aneurisma poplíteo (trombose) $)^{4,15,16}$. Com a maior longevidade da população e a utilização do exame de dúplex arterial de membros inferiores sendo utilizado de forma mais abrangente, é provável que tenhamos mais oportunidades de tratar pacientes com aneurisma de artéria femoral superficial e que estas e outras dúvidas sejam respondidas. Embora os resultados do tratamento cirúrgico sejam satisfatórios (Tabela 1), o advento de técnicas endovasculares, talvez contribua para reduzir a morbidade existente.

\section{Referências}

1. Pappas G, Janes JM, Bernatz PE, et al. Femoral aneurysms. JAMA. 1964;190:97.

2. Bonamigo TP, Ristow A, Buriham E, Cinelli Jr M. Doenças da aorta e seus ramos: diagnóstico e tratamento. São Paulo: Byc; 1991.

3. Ruptured aneurysm of the superficial femoral artery. Scand J Thorac Cardiovasc Surg. 1975;9:181-2.

4. Kremen J, Menzoian JO, Corson JD, Bush HL, LoGerfo FW. Atherosclerotic aneurysms of the superficial femoral artery: a literature review and report of six additional cases. Am Surg. 1981;47:338-42.
5. Ruptured aneurysm of the superficial femoral artery. Panminerva Med. 1995;37:155-8.

6. Aneurysms of the superficial femoral artery at the rupture stage. Apropos 2 personal cases. Minerva Chir. 1991;46:1071-3.

7. Ruptured atherosclerotic aneurysm of the superficial femoral artery. Case report. Acta Chir Scand. 1989;155:493-4.

8. Superficial femoral artery aneurysm with imminent rupture. Wiad Lek. 1989;42:334-6.

9. Galindo CC, Lima CA, Cardoso JE, Galindo Filho G, Costa VS, Penha FM. Aneurisma aterosclerótico isolado da artéria femoral superficial-relato de caso. J Vasc Bras. 2003;2:145-7

10. Aneurysms of the superficial femoral artery: a report of two cases and review of the literature. J Vasc Surg. 1992;16:790-3.

11. Dighe S, Thomas P. Ruptured superficial femoral artery aneurysm treated by simple ligation. Singapore Med J. 2008;49:e151-2.

12. Endoluminal grafting in the treatment of iliac and superficial femoral artery disease. Tex Heart Inst J. 1997;24:185-92.

13. Spontaneous rupture of the superficial femoral artery treated via an endovascular approach. Tex Heart Inst J. 2008;35:66-8.

14. Spontaneous rupture of the superficial femoral artery treated with endovascular stent-grafting. Cardiovasc Intervent Radiol. 2007;30:1016-9.

15. Superficial femoral artery aneurysms: an unusual entity? J Vasc Surg. 2002;36:571-4.

16. Leon Jr LR, Taylor Z, Psalms SB, Mills JL. Degenerative aneurysms of the superficial femoral artery. Eur J Vasc Endovasc Surg. 2008;35:332-40.

17. Hardy DG, Eadie DG. Femoral aneurysms. Br J Surg. 1972;59:614-6.

18. Aneurysm of the superficial femoral artery. Chir Ital. 1984;36:260-5.

19. Mayall JC, Mayall RC, Mayall AC, Mayall LC. Peripheral aneurysms. Int Angiol. 1991;10:141-5.

20. Isolated true atherosclerotic aneurysms of the superficial femoral artery. Case report and literature review. J Cardiovasc Surg (Torino). 1993;34:511-2.

21. Atallah C, al Hassan HK, Neglén P. Superficial femoral artery aneurysm - an uncommon site of aneurysm formation. Eur J Vasc Endovasc Surg. 1995; 10:502-4.

22. Atherosclerotic aneurysms of the superficial femoral artery: report of two ruptured cases and review of the literature. Vasc Med. 1998;3:275-9.

23. Rupture of infected superficial femoral artery aneurysm. A case report. Acta Chir Belg. 2002;102:276-8.

24. Surgical treatment for an atherosclerotic aneurysm of the superficial femoral artery: report of a case. Surg Today. 2004;34:188-9.

25. Rupture of an isolated true superficial femoral artery aneurysm: case report. G Chir. 2005;26:215-7.

26. Rupture of voluminous atherosclerotic superficial femoral artery aneurysm. Chir Ital. 2005;57:661-7.

Correspondência:

Alexandre Faraco de Oliveira

Rua Vidal Ramos Júnior, 164, Centro

CEP 88502-120 - Lages, SC

Tel.: (49) 3224.3872

E-mail: afaraco@gmail.com 